\title{
Educación para la salud en centros penitenciarios: evaluación de una experiencia en personas con diabetes
}

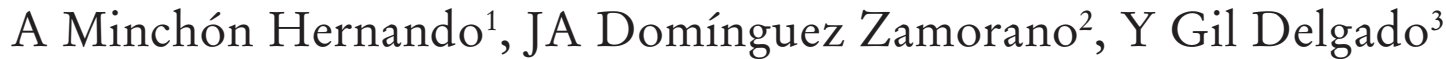

${ }^{1}$ Director de Enfermería del Hospital Infanta Elena (Huelva)

${ }^{2}$ Supervisor de Enfermería del Centro Penitenciario de Huelva

${ }^{3}$ Técnico Superior en Nutrición y Dietética

\section{RESUMEN:}

Fundamento: La estrategia formativa propuesta en el Centro Penitenciario de Huelva pretende dar a los internos diabéticos formación e información básica sobre su enfermedad.

Método: Estudio descriptivo y transversal realizado en el Centro Penitenciario de Huelva. Se recoge información mediante la utilización de cuestionarios diseñados para ello. Se realiza un análisis descriptivo mediante la utilización de medias para variables cuantitativas y frecuencias absolutas y relativas para las variables cualitativas.

Resultados: 27 diabéticos encuestados (24 hombres y 3 mujeres), tasa de participación de 69,2\%, tasa de respuesta 84,4\%, edad media 48,3 años I.C. $95 \%$ (43,7 a 52,9). El 66,7\% insulinodependientes y un 33,3\% en tratamiento con antidiabéticos orales. Tasa de acierto en preguntas que valoran conocimientos adquiridos superior al $80 \%$.

Conclusiones: Se parte de la necesidad de aumentar los conocimientos ante las dificultades que los internos tienen de adecuar las dietas estándar a los requerimientos nutricionales de su enfermedad. Los resultados a tan corto plazo no posibilitan valorar el cambio de actitud si bien se destaca la participación e interés de los internos y la posibilidad de establecer grupos de autoayuda.

Palabras claves: Educación en Salud; Prisiones; Diabetes Mellitus; Alimentación; Nutrición, en Salud Pública; España; Prisioneros; Promoción de la Salud.

\section{HEALTH EDUCATION IN PRISONS: ASSESSMENT OF AN EXPERIENCE WITH DIABETICS}

\section{ABSTRACT:}

Background: A training strategy designed at Huelva Prison sets out to provide diabetic inmates with training and basic information about the illness.

Method: Descriptive and cross-sectional study carried out at Huelva Prison. Information was acquired using questionnaires prepared for the study. A descriptive analysis was then made using averages for quantitative variables and absolute and relative frequencies for the quantitative variables.

Results: 27 interviewed diabetics (24 men and 3 women), participation rate, $69.2 \%$, response rate $84.4 \%$, average age 48.3 CI $95 \%$ (43.7 to 52.9$)$. $66.7 \%$ insulin dependent and $33.3 \%$ receiving oral anti-diabetic treatment. Hit rate in questions assessing knowledge was over $80 \%$.

Conclusions: The study derives from the need to increase awareness of inmates' difficulties in adapting standard diets to the nutritional requirements imposed by the illness. Short term results such as these cannot be used to assess changes of attitude, although one notable conclusion that can be drawn is the high level of participation and interest by inmates and the consequent possibility of setting up self-help groups.

Key words: Health Education; Prisons; Diabetes Mellitus; Feeding; Nutrition, Public Health; Spain; Prisoners; Health Promotion. 


\section{CUADRO DE TEXTO 1: CONTENIDOS DE LAS SESIONES FORMATIVAS}

- Principios básicos de la nutrición

$\sqrt{ }$ Información sobre nutrientes

$\checkmark$ Plan de alimentación para el control de la diabetes

$\checkmark$ Recomendaciones: como el reparto de comidas a lo largo del día

- Plan de alimentación saludable para un diabético $\checkmark$ Eliminar alimentos insalubres

$\sqrt{ }$ Alternativas existentes en el Centro Penitenciario

$\checkmark$ Incluir todos los grupos de alimentos

$\checkmark$ Controlar alimentos ricos en hidratos de carbono

- La importancia del agua

$\checkmark$ Adecuada hidratación junto a la alimentación

$\checkmark$ Bebida de elección en la diabetes

$\sqrt{ }$ Importancia en el desarrollo del ejercicio físico

$\checkmark$ Posibles sustitutos más saludables
- La actividad física y su importancia en el diabético

$\sqrt{ }$ Actividad física adaptada y su importancia en el diabético

$\checkmark$ Beneficios

$\sqrt{ }$ Uso de calzado adecuado

$\checkmark$ Preveer posible hipoglucemia

$\sqrt{ }$ Dieta-ejercicio-tratamiento médico como pilar fundamental

- Beneficios de una alimentación equilibrada para un diabético

$\checkmark$ Ajuste de glucemia

$\checkmark$ Ajuste de dosis adecuada de medicación

$\checkmark$ Importante papel de la fibra en la excreción de la glucosa

$\checkmark$ Prevención enfermedades asociadas a la diabetes

\section{INTRODUCCIÓN}

Las singulares características del entorno penitenciario hacen necesario la utilización de nuevas estrategias dirigidas fundamentalmente a la promoción de la salud y a la prevención de la enfermedad. Entre estas especiales características cabe destacar sobre todo la alta prevalencia de patologías, el bajo nivel socioeconómico y cultural, así como la dificultad de acceso a los servicios sanitarios. Algunos estudios señalan ${ }^{1}$ que las desigualdades sociales pueden haber incrementado la prevalencia de enfermedades crónicas como la DM2 mientras que, por otro lado, la provisión y la calidad de los servicios de atención primaria pueden ser importantes para disminuir el impacto generado por las desigualdades.

La formación en materia de salud a la población reclusa se establece como objetivo primordial entre los responsables de enfermería del Centro Penitenciario de Huelva como centro de atención primaria y su hospital de referencia, el Hospital Infanta Elena. Es en este entorno de colaboración mutua donde, desde hace dos años, se lleva a la práctica un programa dirigido a los internos ${ }^{2}$ que en esta ocasión se orienta, dados los factores anteriormente expuestos, hacia las personas con diabetes. Las condiciones de seguimiento y control dietético y dietoterápico de la cocina de prisiones en general y hacia estos internos en particular, nos hacen plantearnos la formación en ali- mentación, hacia esta población de riesgo como un elemento básico para el control glucémico.

Partimos del conocimiento de que la Educación Alimentaria y Nutricional, dentro del campo de la nutrición humana, en un elemento importante en la prevención, promoción y tratamiento de alteraciones nutricionales y enfermedades crónicas ${ }^{3}$.

La diabetes mellitus es, por el número de personas afectadas y por el coste asociado a la enfermedad y sus complicaciones (una de las primeras causas de mortalidad en España ${ }^{4}$ ) una patología que requiere que los propios enfermos sean educados en el conocimiento del problema y su tratamiento ${ }^{5}$.

Los objetivos que nos planteamos para estas sesiones formativas fueron los siguientes:

- Conocer la base de una alimentación sana.

- Desarrollar hábitos saludables y corregir malos hábitos alimenticios.

- Conocer los alimentos con hidratos de carbono y su frecuencia de consumo.

- Entender la importancia y saludabilidad del agua para un diabético frente a bebidas menos sanas.

- Promover el ejercicio físico como forma de vida saludable en un diabético.

- Prevenir y contrarrestar posibles alteraciones de la glucemia.

- Conocer los beneficios de una alimentación sana para un mejor control de su enfermedad. 
- Prevenir factores de riesgo: colesterol elevado, hiperglucemia, hipertensión arterial, obesidad, etc. mediante la alimentación.

A la finalización de las sesiones decidimos recoger información a través de unos cuestionarios que nos permitieran evaluar tanto el proceso mismo de la intervención educativa como los resultados que dicha intervención hubiera provocado distinguiendo:

1. Valoración de la sesión formativa por los internos. Para ello se hicieron diversas preguntas sobre la metodología usada.

2. Eficacia o grado de aprendizaje obtenido tras la misma.

3. Efectividad o grado de implicación en actividades futuras relacionadas con el control de la alimentación o la modificación del menú para diabéticos.

\section{MATERIAL Y MÉTODO}

Diseño: estudio descriptivo y transversal realizado en el Centro Penitenciario de Huelva.

El proceso de selección de los internos correspondió al mismo centro penitenciario dentro del Programa para el Control de la Diabetes puesto en marcha durante el año 2008. La selección de la muestra a estudio, se realizó entre todos los internos del Centro Penitenciario con diabetes mellitus y que de forma voluntaria quisieron participar.

Este programa que tiene como finalidad conseguir el control autónomo del paciente tanto dentro del centro como tras su puesta en libertad, se rige por las líneas estratégicas establecidas en el Plan Integral de Diabetes de Andalucía ${ }^{6}$.

Fueron seleccionados 39 internos con diabetes tipo I y II que asistieron a exposiciones teóricas de dos horas de duración en grupos de menos de 15 internos, con un total de 3 sesiones, en las que primó la participación activa de los internos para favorecer la resolución de las dudas.

Las horas de exposición se adaptaron al normal devenir de la actividad del centro al objeto de no interferir demasiado en la misma.

Contenidos de las sesiones formativas ${ }^{7-14}$ :

- Principios básicos de la nutrición.

- Plan de alimentación saludable para un diabético.

- La importancia del agua.

- Actividad física y su importancia en el diabético.

- Recomendaciones para prevenir complicaciones.
- Beneficios de la alimentación equilibrada para un diabético.

Los contenidos de las sesiones se detallan de forma más extensa en el cuadro de texto 1 .

Recogida de información: fue realizada mediante unos cuestionarios elaborados con tal fin, una vez finalizada cada una de las charlas.

El análisis estadístico: fue descriptivo, utilizando medias para las variables cuantitativas y frecuencias absolutas y relativas para las variables cualitativas siendo el intervalo de confianza utilizado del $95 \%$.

El análisis de los datos fue realizado mediante el paquete estadístico SPSS v. 12.

Aspectos ético-legales: se solicitó autorización para la realización de este trabajo a la Unidad de Apoyo de la Dirección General de Instituciones Penitenciarias, en los términos legales a los que hace referencia la Orden Circular 7/99 sobre "Trabajos, estudios e investigaciones en el medio penitenciario"15.

A todos los pacientes se les solicitó consentimiento autorizado por escrito, en base a disposiciones legales vigentes, y en concreto en base a la Ley Orgánica 15/1999 de 13 de diciembre, de Protección de Datos de Carácter Personal ${ }^{16}$, así como en los artículos 4.2.b; 211.2 y 211.3 del vigente Reglamento Penitenciario ${ }^{17}$.

\section{RESULTADOS}

El número total de encuestados (27), fue menor que el número de asistentes (32, de un total de 39 diabéticos), presentando, por tanto, una tasa de participación de $69,2 \%$ y una tasa de respuesta del $84,4 \%$ ya que algunos internos tuvieron que abandonarla en diferentes momentos de la exposición por distintos motivos y el haberlos recaptados con posterioridad para las encuestas, hubiera falseado los datos de las mismas, por no disponer los internos, de toda la información necesaria. En ningún momento hubo negativas a participar.

Cumplimentaron los cuestionarios, 24 varones $(88,9 \%)$ y 3 mujeres $(11,1 \%)$, cuya media de edad fue de 48,3 años con un I.C. $95 \%$ (43,7 a 52,9), presentando una edad mediana de 46,5 años y un rango de 32,1 años y 77,5 años.

Con respecto a la diabetes, 18 (66,7\%) seguían tratamiento insulínico y $9(33,3 \%)$ tratamiento con antidiabéticos orales.

En cuanto al país de origen, dos de los encuestados eran extranjeros. Según su procedencia al centro penitenciario, $5(18,5 \%)$ de las personas era su primer 


\begin{tabular}{|c|c|c|}
\hline $\begin{array}{l}\text { ¿Cuáles deberían ser los alimentos eliminados de } \\
\text { una dieta para diabéticos? }\end{array}$ & $\begin{array}{l}\text { lados, pasteles y caramelos } \\
\qquad 22(81,48 \%)\end{array}$ & $\begin{array}{c}\text { Frutas, lácteos y cereales } \\
5(18,52 \%)\end{array}$ \\
\hline ¿Cuál es la alternativa de una coca-cola normal? & $\begin{array}{l}\text { Coca-cola Light o zero. } \\
25(92,59 \%)\end{array}$ & $\begin{array}{c}\text { Vino } \\
2(7,41 \%)\end{array}$ \\
\hline ¿Se aconseja tomar azúcar en el café? & $\begin{array}{c}\text { Sí } \\
0(0,00 \%)\end{array}$ & $\begin{array}{l}\text {, pero se puede sustituir por edulcorantes } \\
\qquad 27(100 \%)\end{array}$ \\
\hline ¿Cuántas comidas se aconseja realizar durante el día? & $\begin{array}{l}3 \text { comidas al día } \\
2(7,41 \%)\end{array}$ & $\begin{array}{l}5 \text { ó } 6 \text { comidas al día } \\
25(92,59 \%)\end{array}$ \\
\hline ¿Es necesario que un diabético realice el desayuno? & $\begin{array}{l}\text { Sí, siempre } \\
27(100 \%)\end{array}$ & $\begin{array}{c}\text { No } \\
0(0,00 \%)\end{array}$ \\
\hline $\begin{array}{l}\text { Ante los siguientes alimentos, ¿cuáles se aconsejan } \\
\text { tomar en un desayuno para un diabético? }\end{array}$ & $\begin{array}{l}\text { Fruta, lácteos y cereales } \\
\qquad 27(100 \%)\end{array}$ & $\begin{array}{l}\text { Leche y un dulce } \\
\quad 0(0,00 \%)\end{array}$ \\
\hline ¿Cuántas frutas se aconseja tomar durante un día? & $\begin{array}{l}1 \text { pieza al día } \\
2(7,41 \%)\end{array}$ & $\begin{array}{l}3 \text { piezas al día } \\
25(92,59 \%)\end{array}$ \\
\hline ¿Cuánta verdura se aconseja al día? & $\begin{array}{c}1 \text { ración } \\
2(7,41 \%)\end{array}$ & $\begin{array}{l}2 \text { raciones } \\
25(92,59 \%)\end{array}$ \\
\hline $\begin{array}{l}\text { De los siguientes alimentos, ¿cuáles son de rápida } \\
\text { absorción (consumo poco frecuente)? }\end{array}$ & $\begin{array}{c}\text { Frutas } \\
14(51,85 \%)\end{array}$ & $\begin{array}{c}\text { Repostería, azúcares y golosinas } \\
13(48,15 \%)\end{array}$ \\
\hline $\begin{array}{l}\text { De los siguientes alimentos, ¿cuáles son de absorción } \\
\text { lenta (consumo diario)? }\end{array}$ & $\begin{array}{c}\text { Cereales } \\
26(96,3 \%)\end{array}$ & $\begin{array}{l}\text { Pasteles } \\
1(3,7 \%)\end{array}$ \\
\hline ¿Qué bebida es la más adecuada para un diabético? & $\begin{array}{l}\text { Refrescos } \\
0(0,00 \%)\end{array}$ & $\begin{array}{c}\text { Agua } \\
27(100 \%)\end{array}$ \\
\hline La actividad física, ¿la pueden realizar todos los diabéticos & $\begin{array}{cc}\text { cos? } & \text { Sí } \\
& 27(100 \%) \\
\end{array}$ & $\begin{array}{c}\text { No } \\
0(0,00 \%)\end{array}$ \\
\hline ¿Existe algún pilar fundamental para los diabéticos? & $\begin{array}{l}\text { No existe ninguno } \\
2(7,41 \%)\end{array}$ & $\begin{array}{l}\text { Dieta-ejercicio-medicación (o insulina) } \\
\qquad 25(92,59 \%)\end{array}$ \\
\hline $\begin{array}{l}\text { Antes de realizar un ejercicio, ¿sería aconsejable tomar } \\
\text { hidratos de carbono? }\end{array}$ & $\begin{array}{c}\text { Sí } \\
23(85,19 \%)\end{array}$ & $\begin{array}{c}\text { No } \\
4(14,81 \%)\end{array}$ \\
\hline $\begin{array}{l}\text { Si un diabético se pone más insulina de la que } \\
\text { debiera, ¿qué se debería hacer? }\end{array}$ & $\begin{array}{l}\text { No pasa nada, no es tan } \\
\text { importante } \\
2(7,41 \%) \\
\end{array}$ & $\begin{array}{c}\text { Aumentar la ingesta de alimentos } \\
\text { farináceos, fruta o leche } \\
25(92,59 \%)\end{array}$ \\
\hline $\begin{array}{l}\text { ¿Crees que se pueden tener complicaciones futuras } \\
\text { por una mala alimentación? }\end{array}$ & $\begin{array}{c}\text { Sí } \\
26(96,3 \%)\end{array}$ & $\begin{array}{c}\text { No } \\
1(3,7 \%)\end{array}$ \\
\hline $\begin{array}{l}\text { ¿Crees que hay alimentos apropiados para diabéticos } \\
\text { en el economato de tu módulo? }\end{array}$ & $\begin{array}{c}\text { Sí } \\
3(11,11 \%)\end{array}$ & $\begin{array}{c}\text { No } \\
24(88,89 \%)\end{array}$ \\
\hline $\begin{array}{l}\text { ¿Sabías qué tipo de alimentación era la correcta antes } \\
\text { de esta charla? }\end{array}$ & $\begin{array}{c}\text { Sí } \\
17(62,96 \%)\end{array}$ & $\begin{array}{c}\text { No } \\
10(37,04 \%)\end{array}$ \\
\hline
\end{tabular}

Tabla 1: Grado de aprendizaje obtenido tras la charla.

ingreso, y las 22 restantes $(81,5 \%)$ ya estaban en prisión o provenían de otros centros.

Los datos obtenidos de los cuestionarios sobre grado de aprendizaje se adjuntan en la Tabla 1 . Los datos sobre la valoración realizada por los internos sobre la charla se adjuntan en la Tabla 2, y los datos sobre el grado de implicación en futuras actividades se adjuntan en la Tabla 3.
Como datos destacables señalaremos el alto grado de respuestas correctas dentro de las preguntas que pretenden medir el grado de aprendizaje obtenido tras la sesión formativa, existiendo una única pregunta en la que la tasa de acierto se haya visto comprometida con un $51,85 \%$ (pregunta referente a los alimentos de rápida absorción). El 88,88\% de las preguntas que pretendían valorar los conocimientos adquiridos 


\begin{tabular}{lcc}
\hline & Sí & No \\
\hline$¿$ ¿Ha aprendido cosas interesantes en esta charla? & $27(100 \%)$ & $0(0,00 \%)$ \\
\hline$¿$ Cree que puede mejorar el control de su enfermedad después de esta charla? & $27(100 \%)$ & $0(0,00 \%)$ \\
\hline$¿$ Cree que la charla ha sido corta? & $14(51,85 \%)$ & $13(48,15 \%)$ \\
\hline ¿Le ha gustado la forma en la que se ha impartido la charla? & $27(100 \%)$ & $0(0,00 \%)$ \\
\hline
\end{tabular}

Tabla 2: Valoración de la charla por los internos.

\begin{tabular}{|c|c|c|}
\hline & Sí & No \\
\hline ¿Cree que sabe lo suficiente sobre la diabetes? & $10(37,04 \%)$ & $17(62,96 \%)$ \\
\hline $\begin{array}{l}\text { ¿Cree que va a aplicar en adelante los conocimientos que ha adquirido } \\
\text { en esta charla? }\end{array}$ & $26(96,3 \%)$ & $1(3,7 \%)$ \\
\hline ¿Le gustaría acudir a más charlas de este tipo? & $27(100 \%)$ & $0(0,00 \%)$ \\
\hline $\begin{array}{l}\text { ¿Cree que le ayudaría a mejorar el control de su enfermedad si tuviera más } \\
\text { conocimientos de alimentación para diabéticos? }\end{array}$ & $27(100 \%)$ & $0(0,00 \%)$ \\
\hline ¿Estaría de acuerdo en tener charlas nutricionales de forma periódica? & $26(96,3 \%)$ & $1(3,7 \%)$ \\
\hline $\begin{array}{l}\text { ¿Estaría dispuesto a acudir a una consulta de nutrición con frecuencia para que } \\
\text { le enseñara los alimentos que debe tomar y con ello mejorar su enfermedad? }\end{array}$ & $26(96,3 \%)$ & $1(3,7 \%)$ \\
\hline ¿Cree que la dieta para diabéticos es adecuada en este Centro? & $5(18,52 \%)$ & $22(81,48 \%)$ \\
\hline ¿Cree que podría adaptarse mejor la dieta a los diabéticos? & $26(96,3 \%)$ & $1(3,7 \%)$ \\
\hline
\end{tabular}

Tabla 3: Implicación en actividades futuras relacionadas con el control de la alimentación o la modificación del menú para diabéticos.

tuvieron una tasa de acierto de más de un $80 \%$ de los asistentes.

Mención expresa merece la valoración positiva que los oyentes realizan de la sesión, donde penalizan únicamente la duración de la misma, con un $51,85 \%$ que piensan que no les ha parecido corta. Este dato nos hace replantearnos la elaboración de actividades futuras, en donde deben ser incluidos quizás otros elementos pedagógicos o audiovisuales que hagan más amena la exposición, habida cuenta que el $100 \%$ de los asistentes aprueban la forma en la que se celebraron las sesiones y su utilidad.

En el apartado referente a la efectividad destacamos el alto índice de compromiso obtenido para implicarse en acciones futuras de esta índole, con un compromiso inicial mayor del $95 \%$ de los asistentes. Queremos resaltar la respuesta a la pregunta sobre la idoneidad de la dieta para diabéticos del Centro, que ha ofrecido un $81,48 \%$ de respuestas negativas, frente al $96.3 \%$ que piensa que puede adaptarse mejor a las necesidades marcadas por su enfermedad.

\section{DISCUSION}

La realización de charlas-taller en grupos reducidos a colectivos marginales o con un bajo nivel de conocimiento, sobre temas que les afectan de forma muy especial como es su salud, es un buen medio para crear aprendizaje significativo que les permita un mejor control de su enfermedad. Los estudios de Cerrillo $^{18,19}$ y Núñez ${ }^{20}$ sobre la eficacia de la educación diabetológica grupal frente a la individual centran el debate en la validez de ambos modelos de prácticas avanzadas en la enfermería actual, orientando esta actividad hacia intervenciones estructuradas, planificadas, en una secuencia lógica y, por último, común a todos los profesionales que, de una u otra forma, intervienen en ella. En nuestro contexto asistencial parece demostrarse igualmente que estas actividades grupales son, frente a las actividades individuales, más eficientes y efectivas $y$, por ende, tal como refiere $\mathrm{Du}$ ro Martínez ${ }^{21}$, generadoras de más satisfacción personal entre los profesionales. 
Con las encuestas hemos demostrado de forma evidente que se han conseguido los objetivos de mejorar el grado de conocimiento que estos enfermos tienen sobre el manejo de su enfermedad y con ello disminuir los riesgos de complicaciones características de esta patología.

Para el buen manejo de la diabetes es básico el conocimiento de la correcta alimentación que este colectivo debe tener y del que carece en la práctica totalidad de los casos.

Es significativo que no exista prácticamente nada referente a educación nutricional a diabéticos en prisión teniendo en cuenta la importantísima relación que existe entre alimentación correcta y el adecuado manejo de la diabetes, más si cabe en colectivos marginales con un seguimiento sanitario ocasional en el exterior.

Hubiera sido más plausible pasar la encuesta a un grupo control, pero debido al pequeño grupo de diabéticos que coexisten a la vez en un Centro Penitenciario (39 en nuestro caso) y la dificultad para repetir acciones coordinadas de este tipo en un futuro se desestimó la idea.

Igualmente se nos planteó pasar las encuestas antes y después de la exposición para una mejor valoración de los resultados, pero ello suponía alargar cada una de las sesiones con el cansancio y la merma en la atención que hubiera conllevado. Hubiera podido suponer por el contrario, una pérdida de encuestados ya que, tal y como se presenta en los resultados, entre las 3 sesiones de 2 horas cada una de ellas se perdieron 5 encuestados al tener algunos internos que abandonar las sesiones por diferentes motivos regimentales sin finalizar las mismas.

Somos conscientes de lo limitado de la muestra que artefacta en cierto sentido los resultados, pero hay que señalar que para las charlas fueron seleccionados todos los diabéticos del Centro, aunque algunos no pudieron asistir o, como hemos mencionado, la tuvieron que abandonar antes de su finalización por motivos regimentales que escapan a nuestro control. Sin embargo, señalaremos que las encuestas fueron realizadas por todos los internos que finalizaron las charlas, no existiendo ningún caso de rechazo a la colaboración en el estudio.

Otras limitaciones: Entre las respuestas discordantes o erróneas hay que contemplar la existencia de un interno con problemas de hipoacúsia severa que reconoció no haber oído mucho de las sesiones formativas, algunos internos con problemas de falta de concentración que incidieron negativamente en el relleno de la encuesta tras casi dos horas de explicación, o la existencia de internos con tratamiento oral úni- camente, que hizo que respondieran mal a la pregunta relacionada con qué hacer tras una dosis mayor de insulina de la debida por no tener conocimiento del tema. Se observó un interno poco comprometido con la enfermedad y que se reconoce totalmente desinteresado en la corrección de hábitos (aún a sabiendas de que lo son), y en sesiones posteriores no sería susceptible, en principio, de trabajar con el resto del grupo.

Entre nuestros objetivos de futuro está el repetir la encuesta en un plazo aproximado de 3 meses, extendiendo la experiencia en el tiempo, e incluso pudiendo intervenir en la realización del menú para los diabéticos del Centro atendiendo a las especiales características que este colectivo precisa, tanto en cuanto a la elaboración del mismo como en el reparto de las calorías diarias a lo largo del día.

Debido a los resultados obtenidos, la buena aceptación de estas sesiones por parte de los internos y los ponentes, y la experiencia obtenida, podemos plantearnos nuevas actividades y el desarrollo de programas de intervención más ambiciosos que mejoren la salud y calidad de vida de nuestros pacientes, la creación de una red de educadores entre profesionales de ambos centros y promover la incorporación de los internos como educadores-orientadores en programas de salud.

\section{CORRESPONDENCIA}

José Antonio Domínguez Zamorano

Centro Penitenciario de Huelva

Ctra. La Ribera, s/n.

21610 Huelva.

E-mail: jayosevali@hotmail.com

\section{BIBLIOGRAFÍA}

1. Ruiz-Ramos M, Escolar-Pujolar A, Mayoral-Sánchez E, Corral-San Laureano F, Fernández-Fernández I. La diabetes mellitus en España: mortalidad, prevalencia, incidencia, costes económicos y desigualdades. Gac Sanit 2006; 20 Supl 1: 15-24

2. Minchón Hernando A, Domínguez Zamorano JA. Aportación de enfermería al proceso de Educación para la Salud en Instituciones Penitenciarias. Documentos de Enfermería 2008; 29: 18-20.

3. Fuillerat Alfonso R. Psicología y nutrición en el desarrollo ontogenético en la edad infanto-juvenil. Nutr. Hosp. [revista en internet]. 2004 Jul. [citado el 02 de noviembre de 2008]; 19(4): 209-224. 
Disponible en: http://scielo.isciii.es/scielo.php? script=sci_arttext\&pid=S0212-161120040004000 03\&lng=es.

4. España 2006. Mortalidad por Causa, Sexo y Grupo de edad (CIE 10). Tasa por $100.000 \mathrm{~h}$ y Mortalidad proporcional. Disponible en: http://www. isciii.es/htdocs/centros/epidemiologia/anexos/ww01_ed.htm

5. Selli Lucilda, Papaléo Ledi Kauffmann, Meneghel Stela Nazareth, Torneros Julián Zapico. Técnicas educacionales en el tratamiento de la diabetes. Cad. Saúde Pública [revista en internet]. 2005 Oct. [citado el 02 de noviembre de 2008]; 21(5): 1366-1372. Disponible en: http://www.scielo.br/ scielo.php?script=sci_arttext\&pid=S0102-311X $2005000500008 \& \operatorname{lng}=$ en.

6. Plan Integral de Diabetes de Andalucía: 20032007. $10^{a}$ ed. Sevilla: Consejería de Salud, 2003

7. Mataix Verdú, J. Tabla de Composición de Alimentos. $4^{a}$ ed. Granada: Universidad de Grana$\mathrm{da} ; 2003$.

8. Asociación de Enfermería. Educación para la Salud: Alimentación y Nutrición. Valencia: La Sirena; 2005.

9. Figuerola D. Alimentación y Diabetes. Barcelona: De Masson; 2003.

10. Larrañaga Coll IJ, Carballo Fernández M, Rodríguez Torres MM, Fernández Sainz JA. Dietética y Dietoterapia. $1^{\text {a }}$ ed. Madrid. McGrawHill/Interamericana de España S.A; 1997.

11. Junta de Andalucía; Consejería de Salud. Plan para la Promoción de Actividad Física y la Alimentación Equilibrada, 2004-2008. Sevilla; 2004.

12. Anguita C, Caixás A, Clapés J, Claveria M, Gallach I, Jansà M, et al. Documento de Consenso sobre Recomendaciones Nutricionales y de Educación Alimentaria en la Diabetes. $1^{\mathrm{a}}$ ed. Barcelona: Asociaciò Catalana de Diabetis; 2003.

13. Jansá M, Vidal M, Gomis R, Esmatjes E. La diabetes. Barcelona: Forunclinic/ Fundación BBVA. Hospital Clinic de Barcelona; 2006.

14. Controlar la Diabetes. Comer Sano con la Fundación Grande Covián y las Recetas de Karlos Arguiñano. $1^{a}$ ed. Bilbao: Bainet Media S.A.; 2007.

15. Trabajo, estudios e investigaciones en el medio penitenciario. Dirección General de Instituciones
Penitenciarias. Circular C 07/99 IP de 8 de julio. [citado el 02 de noviembre de 2008] Disponible en: http://www.mir.es/INSTPEN/INSTPENI/ Archivos/c-1999-07.pdf

16. LEY ORGÁNICA 15/1999, de 13 de diciembre, de Protección de Datos de Carácter Personal (B.O.E. de 14-12-99)

17. REAL DECRETO 190/1996, de 9 de febrero, por el que se aprueba el Reglamento penitenciario (B.O.E. de 15-02-96)

18. Cerrillo Patiño J. La educación grupal a pacientes diabéticos tipo 2 mejora los conocimientos de la enfermedad, el control metabólico y factores de riesgo cardiovascular [Dalmau Llorca MR, García Bernal G, Aguilar Martín C y Palau Galindo A. Educación grupal frente a individual en pacientes diabéticos tipo 2. Aten Primaria 2003; 32(1): 36-41] Evidentia. 2006 nov-dic. [citado el 07 de julio de 2009]; 3(12). Disponible en: http:// indez-f.com/evidentia/n12/271articulo.php.

19. Cerrillo Patiño J. Ocho talleres de 1 hora realizados a pacientes diabéticos tipo 2 mejoran el control metabólico durante un año de seguimiento [González Marcos M, Perpinyá D, Mir S, Casllas P, Melció D y García MT. Efectividad de un programa de educación grupal estructurada en personas con diabetes mellitus tipo 2. Enferm clínica. 2005; 15(3): 141-6] Evidentia. 2006 nov-dic. [citado el 07 de julio de 2009]; 3(12). Disponible en: http://indez-f.com/evidentia/n12/272articulo. php.

20. Núñez Feria R. ¿Mejora el manejo del régimen terapéutico y el control metabólico de los diabéticos tipo II que acuden a talleres de educación sanitaria grupal? Evidentia. 2006 nov-dic. [citado el 07 de julio de 2009]; 3(12). Disponible en: http://indez-f.com/evidentia/n12/277articulo. php.

21. Duro Martínez Juan Carlos. El discurso de los profesionales de atención primaria de la Comunidad de Madrid sobre el trabajo con grupos: sentido, finalidades y ámbitos de intervención. Rev. Esp. Salud Pública [revista en internet]. 2002 Oct; [citado el 02 de noviembre de 2008]; 76(5): 545559. Disponible en: http://scielo.isciii.es/scielo. php? script $=$ sci_arttext\&pid $=$ S1135-5727200200 0500015\&lng=es. 